\title{
N-Propargyl Caffeamide Skews Macrophages Towards a Resolving M2-Like Phenotype Against Myocardial Ischemic Injury via Activating Nrf2/HO-1 Pathway and Inhibiting NF-kB Pathway
}

\author{
Yuanyuan Chenga Chuanbin Yang ${ }^{a} \quad$ Dan Luo $^{a} \quad$ Xuechen Lib $\quad$ X. Chris Le \\ Jianhui Rong a \\ aSchool of Chinese Medicine, Li Ka Shing Faculty of Medicine, The University of Hong Kong, Hong \\ Kong, bepartment of Chemistry, Faculty of Science, University of Hong Kong Hong Kong, China, \\ 'Department of Laboratory Medicine and Pathology, Faculty of Medicine and Dentistry, University of \\ Alberta, Edmonton, Alberta, Canada
}

\section{Key Words}

Myocardial infarction - Macrophage polarization - $\mathrm{N}$-propargyl caffeamide (PACA) - Inducible nitric oxide synthase (iNOS) • Heme oxygenase-1 (HO-1)

\begin{abstract}
Background/Aims: Macrophages exhibit dynamic pro-inflammatory and resolving activities in myocardial infarction. The present study investigated whether caffeic acid derivatives could induce macrophage polarization towards a resolving M2 phenotype against myocardial infarction injury. Methods: Western blotting, RT-PCR and flow cytometry techniques are used to evaluate macrophage biomarkers expression and specific proteins in the related signaling pathways. Ligation of the left anterior descending artery induced rat model of myocardial infarction, TTC staining and immunohistochemical staining are used to examine cardioprotective effect in vivo. Results: We initially evaluated the anti-inflammatory activity of four caffeic acid derivatives including n-propargyl caffeamide (PACA) in RAW264.7 macrophages. As result, PACA selectively suppressed the up-regulation of inducible nitric oxide synthase (iNOS) over cyclooxygenase-2 (COX-2) in lipopolysaccharides (LPS)-stimulated cells. We subsequently examined the effects of PACA on macrophage polarization by determining macrophage biomarkers. PACA down-regulated M1 biomarkers (e.g., iNOS, tumor necrosis factor- $\alpha$ (TNF- $\alpha$ ), C-X-C motif chemokine 10 (CXCL10) and CD80) but upregulated M2 biomarkers (e.g., Ym-1 and arginase-1). On the other hand, PACA suppressed macrophage chemotaxis while enhanced macrophage phagocytosis. We further examined the in vivo cardioprotective activity of PACA in a rat model of myocardial infarction. Following

Y. Cheng and C. Yang contributed equally to this work.

\begin{tabular}{ll}
\hline Jianhui Rong & School of Chinese Medicine, Li Ka Shing Faculty of Medicine, The University of Hong Kong \\
& Hong Kong (China) \\
& E-Mail jrong@hku.hk
\end{tabular}
\end{abstract}


ligation of the left anterior descending artery, PACA treatment effectively reduced myocardial infarct size and promoted macrophage M2 polarization. We finally explored the underlying mechanisms. We found that PACA attenuated LPS-induced NF-KB activation while activated Nrf2/HO-1 pathway. HO-1 inhibitor SnPP attenuated the effects of PACA on iNOS expression in LPS-challenged macrophages, possibly by regulating the cross-talk between $\mathrm{HO}-1$ and NFKB pathways. Conclusions: The key finding from the present study was that PACA promoted timely switch of macrophage phenotypes from pro-inflammatory $M 1$ to resolving M2. We anticipate that PACA is a potential drug candidate for the resolution of inflammation and cardiac repair after myocardial infarction.

(C) 2018 The Author(s)

Published by S. Karger AG, Basel

\section{Introduction}

Myocardial infarction causes ischemic necrosis of heart tissues, a leading cause of human death [1]. Upon infarction, proinflammatory immune cells especially polymorphonuclear leukocytes/monocytes are rapidly recruited to the hearts. Infiltrating neutrophils and macrophages are activated to induce inflammatory responses, oxidative stress, and uncontrolled deposition of extracellular matrix components [2]. Depending on the pathological conditions, macrophages undergo dynamic M1/M2 polarization in infarcted hearts [3]. Proinflammatory M1 macrophages produce a battery of proinflammatory mediators including interleukin 6 (IL-6) and tumor necrosis factor- $\alpha$ (TNF- $\alpha$ ) [4]. Macrophages also exhibit an anti-inflammatory and resolving M2 phenotype for resolving post-infarct inflammation and repairing cardiac tissues [4, 5]. The phenotypes of macrophage are defined by the expression pattern of specific biomarkers [4]. Inducible nitric oxide synthase (iNOS) is well-recognized as a key M1 biomarker and induced by several pro-inflammatory cytokines and lipid mediators [6]. In response to inflammatory stimuli, nuclear factor-kappa B (NF- $\kappa$ B) pathway is activated to up-regulate iNOS expression, leading to the overproduction of toxic NO [7]. In contrast, M2 macrophages express lower level of iNOS but higher level of arginase-1 (Arg1) [8]. The iNOS/Arg1 ratio is widely used to define macrophage M1 or M2 polarization. Nevertheless, timely switch from M1 to M2 macrophage profile is critical for the resolution of inflammation and the recovery of cardiac functions in myocardial infarction [9].

Macrophage differentiation and plasticity are under the control of the microenvironment within the pathological contexts [10]. It is now known that pharmacological interventions with antioxidant and anti-inflammatory agents promote M2 macrophage polarization $[11,12]$. Interestingly, heme oxygenase-1 (HO-1) is often activated to provide endogenous persistence against oxidant, inflammatory and immunomodulatory stimuli [13]. H0-1 catalyzes the degradation of pro-oxidant heme into three important antioxidant products, bilirubin, carbon monoxide (CO) and ferrous ion. These products activate different mechanisms to contribute to the pharmacological activities of HO-1 [13, 14]. Consequently, HO-1 activation inhibits mast cell degranulation and cytokine synthesis in several inflammatory disease models $[15,16]$. By mimicking HO- 1 activation, CO-releasing molecules (CORMs) was recently evaluated for anti-inflammatory potential in different in vitro and in vivo models [17]. Along this line, we attempted to demonstrate the pharmacological activation of HO-1 pathway as a new anti-inflammatory strategy [18-20].

Natural products represent a rich source for the discovery of anti-inflammatory agents and immunomodulators [21]. As an example, caffeic acid is widely present in a variety of fruits and medicinal plants [22]. Caffeic acid and its derivatives (e.g., caffeic acid phenethyl ester (CAPE) and octyl caffeate) have anti-oxidant, anti-inflammatory, neuroprotective and anti-cancer activities [23]. Recent studies suggest that caffeic acid and its derivatives have cardioprotective potential against myocardial infarction injury [24, 25]. However, it remains elusive whether and how caffeic acid derivatives affect the functions of macrophages. 
Cheng et al.: Targeting Macrophages for Resolution of Inflammation

In the present study, we investigated a small panel of caffeic acid derivatives including in-house synthesized n-propargyl caffeamide (PACA) and n-propyl caffeamide (PCA) for the potential to regulate macrophage M1/M2 polarization in a rat model of myocardial infarction. We also investigated the potential underlying mechanisms by focusing on the Nrf2/HO-1 and NF- $\kappa B$ pathway.

\section{Materials and Methods}

\section{Chemicals and reagents}

PACA and N-propyl caffeamide (PCA) were synthesized and characterized by NMR and MS as a previous method [19, 26]. Propyl caffeate (PCE) was synthesized by Hangzhou Yuhao chemical Technology Co., Ltd (Hangzhou, China). Antibodies against extracellular-signal-regulated kinase 1/2 (ERK1/2), p38, c-Jun N-terminal kinase (JNK), phospho-ERK1/2, phospho-p38, phospho-JNK, iNOS, cyclooxygenase-2 (COX-2) and glyceraldehydes-3-phosphate dehydrogenase (GAPDH) were obtained from Cell Signaling Technology (Boston, MA, USA). HO-1 antibody was purchased from Enzo Life Sciences (Farmingdale, New York, USA). The antibodies against Nrf2, NF- $\mathrm{KB}$ p65 subunit, Arg1, ED2 and lamin B were obtained from Santa Cruz Biotechnology (Santa Cruz, CA, USA). Antibody against CD68 and ED1 were purchased from Abcam (Cambridge, Massachusetts, USA). FITC-labeled anti-mouse CD80 antibody was obtained from BD biosciences (BD Biosciences, San Diego, CA, USA). Alexa Fluor 594-conjugated goat anti-rabbit IgG antibodies and Alexa Fluor 488-conjugated goat anti-mouse IgG antibodies were purchased from Life technologies (Carlsbad, CA, USA). HRP-conjugated anti-rabbit IgG secondary antibody and other chemicals were obtained from Sigma-Aldrich (St. Louis, MO, USA).

\section{Cell culture and drug treatment}

Murine macrophage cell line RAW264.7 was maintained in Dulbecco's Modified Eagle Medium (DMEM) containing 10\% fetal bovine serum (FBS) and 1\% penicillin/streptomycin (Invitrogen, Carlsbad, CA, USA) at $37^{\circ} \mathrm{C}$ in a $5 \% \mathrm{CO}_{2}$ humidified atmosphere. For drug treatment, the cells at $70-80 \%$ confluence in the complete growth medium were treated with caffeic acid derivatives at the indicated concentrations for specified times, whereas the control cells were treated with equal volume of dimethyl sulfoxide (DMSO) under the same conditions. For Western blot analysis, the cells were pre-treated with drugs for $2 \mathrm{~h}$ and cotreated with LPS for another $24 \mathrm{~h}$.

\section{Measurement of cell viability}

The cell viability was evaluated by a standard colorimetric assay using 3-[4, 5-dimethylthiazol-2-yl]-2, 5-diphenyltetrazolium bromide (MTT) as previously described [27]. Briefly, the cells were treated with caffeic acid derivatives at the indicated concentrations for the indicated times. Cell culture medium was removed, and subsequently the cell monolayers were incubated with $0.5 \mathrm{mg} / \mathrm{ml}$ MTT in phosphate-buffered saline (PBS) for $4 \mathrm{~h}$. The purple formazan product was dissolved in $100 \mu \mathrm{l}$ of DMSO and measured for the absorbance at $570 \mathrm{~nm}$ on a Bio-Rad microplate reader (Hercules, CA, USA). The cell viability was presented as a percentage to that of vehicle-treated controls.

\section{Reverse transcription polymerase chain reaction (RT-PCR) analysis}

Following the treatment with caffeic acid derivatives, the total RNAs were isolated with TRIzol reagent following the manufacture's instruction (Invitrogen, CA, USA). The cDNAs were prepared using SuperScript III reverse transcriptase and oligo primers (Thermo, Waltham, MA, USA) as previous described [28]. The primers for mouse gene were listed as follows:
iNOS:
forward, 5'-AAAGTGACCTGAAAGAGGAAAAGGA-3'
reverse, 5'-TTGGTGACTCTTAGGGTCATCTTGTA-3';
Arg1: forward, $5^{\prime}$-CTCCAAGCCAAAGTCCTTAGAG-3'
reverse, 5'-AGGAGCTGTCATTAGGGACATC-3'
Ym-1: forward, 5'-CATGAGCAAGACTTGCGTGAC-3'
reverse, 5'-GGTCCAAACTTCCATCCTCCA-3'
TNF- $\alpha$ : forward, 5'-ATGAGCACAGAAAGCATG-3' 


\section{Cellular Physiology Cell Physiol Biochem 2018;47:2544-2557

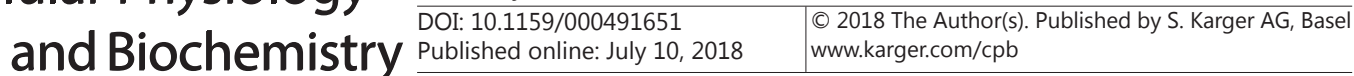 \\ Cheng et al.: Targeting Macrophages for Resolution of Inflammation}

CXCL10: forward, 5'-TCTGAGTGGGACTCAAGGGAT-3'

reverse, 5'-TCGTGGCAATGATCTCAACACG-3'

-actin: forward, 5'-ATGGATGACGATATCGCTGC-3'

reverse, 5'-TTCTGACCCATTCCCACCATC-3'

PCR amplifications were performed under the control of the following program: 1) denaturation, $94^{\circ} \mathrm{C}$ for $3 \mathrm{~min}$; 2) reactions, 30 cycles of $94^{\circ} \mathrm{C}$ for $30 \mathrm{sec}, 60^{\circ} \mathrm{C}$ for $30 \mathrm{sec}$, and $72^{\circ} \mathrm{C}$ for $1 \mathrm{~min}$; ) extension, $72^{\circ} \mathrm{C}$ for 10 min. PCR products were analyzed by gel electrophoresis in $2 \%$ agarose containing GelRed nucleic acid stain (Biotium, Hayward, CA, USA) and visualized under a UV light illuminator.

\section{Western blot analysis}

Following drug treatment, the cellular proteins were prepared and analyzed by Western blotting analysis for protein expression as previously described [29]. Briefly, the cellular proteins were prepared and resolved by electrophoresis on $10 \%$ SDS-polyacrylamide gels and transferred onto a polyvinylidenedifluoride (PVDF) membrane. After overnight incubation in 5\% BSA in Tris-buffered saline with 0.1\% Tween-20 (TBST), the membranes were probed with specific primary antibodies, and subsequently detected by goat anti-rabbit or goat anti-mouse IgG-HRP conjugate secondary antibodies. The activity of peroxidase retained on the blots was visualized with enhanced chemiluminescence (ECL) detection reagents from GE Healthcare (Uppsala, Sweden) according to the manufacturer's instruction.

\section{Cell immunofluorescence staining}

Upon the completion of drug treatment, the cells were fixed with 4\% paraformaldehyde in PBS for 30 minutes, permeabilized with $0.5 \%$ Triton X-100 for 10 minutes and blocked in $5 \%$ normal goat serum in PBS for $1 \mathrm{~h}$ at room temperature. The cells were probed with anti-Nrf2 or anti-p65 antibodies, and subsequently detected by Alexa Fluor 594-conjugated goat anti-rabbit IgG secondary antibody. Following staining the cell nuclei with DAPI, the immunofluorescent images were captured on a Zeiss fluorescence microscopy (Jena, Germany).

\section{Measurement of NO level}

The production of intracellular NO was evaluated by staining with DAF-FM diacetate from Invitrogen (Eugene, OR, USA) according to the manufacturer's instruction. Briefly, at the end of drug treatment, the cells were incubated with DAF-FM diacetate. The images were captured under a Zeiss fluorescence microscope (Jena, Germany). The intracellular NO levels were presented as a percentage to that of the vehicle controls using NIH Image J software (http://imagej.nih.gov/ij/).

\section{Flow cytometry}

RAW 264.7 macrophages were pretreated with $10 \mu \mathrm{M}$ PACA, and then co-stimulated by LPS $(1 \mu \mathrm{g} / \mathrm{mL})$ and IFN- $\gamma(0.5 \mu \mathrm{g} / \mathrm{mL})$ for $24 \mathrm{~h}$. After the treatment, the cells were collected and stained with fluorescenceconjugated marker CD80. Flow cytometric analysis was carried out on a BD LSR Fortessa Analyzer (BD Biosciences, San Jose, CA). The results were analyzed with FlowJo software (Tree Star, Ashland, OR).

\section{Assay of macrophage chemotaxis}

Macrophage chemotaxis was measured in a 12-well Transwell plate according to the manufacturer's introduction [30]. RAW264.7 cells were treated with vehicle, LPS and LPS plus caffeic acid derivatives for 24 $\mathrm{h}$. To generate the conditioned medium, H9c2 cells were challenged under OGD conditions for $12 \mathrm{~h}$. The cell culture medium was recovered as the OGD-conditioned medium, and subsequently transferred to the lower chamber of Transwell plate [31]. Drug-treated RAW264.7 cells were added to the upper chamber $\left(10^{5}\right.$ cells/ well), and allowed to transmigrate over a period of $4 \mathrm{~h}$. The cells that migrated through a polycarbonate membrane with a pore size of $5 \mu \mathrm{m}$ were fixed by methanol for $20 \mathrm{~min}$. The cells in the insert were removed by wiping the microporous membrane with cotton swab. The membranes were then stained with $0.1 \%$ crystal violet for $15 \mathrm{~min}$ and washed with PBS. Finally, the membranes were carefully placed on the glass slide for microscopy observation and imaging. At the end of imaging, the membranes were incubated with $30 \%$ glacial acetic acid for the quantification of macrophages at the wavelength of $570 \mathrm{~nm}$. 


\section{Cellular Physiology Cell Physiol Biochem 2018;47:2544-2557 \begin{tabular}{l|l} 
and Biochemistry Published online: July 10, 2018 & $\begin{array}{l}\text { (c) 2018 The Author(s). Published by S. Karger AG, Basel } \\
\text { www.karger.com/cpb }\end{array}$ \\
\hline
\end{tabular}}

\section{Phagocytosis of latex beads and apoptotic H9c2 cells}

For phagocytosis of latex beads, FluoSpheres ${ }^{\circledR}$ carboxylate-modified beads with the particle size of $1.0 \mu \mathrm{m}$ (Life Technology, NY, USA) were pre-incubated with $10 \%$ FBS/PBS for $1 \mathrm{~h}$ at $37{ }^{\circ} \mathrm{C}$, and then added into RAW264.7 cell culture at 10:1 ratio. Phagocytosis was assessed over a period of $1.5 \mathrm{~h}$. Non-adherent beads were washed away by cold-PBS, whereas the cells were stained with CellTracker, Orange CMTMR probe (Life Technology, NY, USA). For phagocytosis of apoptotic H9c2 cells, H9c2 cells were challenged under OGD conditions for $12 \mathrm{~h}$ for the induction of cell apoptosis. Apoptotic H9c2 cells were labeled with CellTracker,Green CMTMR probe (Life Technology, NY, USA) and subsequently incubated with RAW264.7 macrophages at $10: 1$ ratio at $37^{\circ} \mathrm{C}$ for another $1.5 \mathrm{~h}$. After non-adherent cells were removed, RAW 264.7 cells were stained with CellTracker, Orange CMFDA probe (Life Technology, NY, USA). All slides were examined on a Zeiss laser scanning microscope (Carl-Zeiss, Jena, Germany).

\section{Induction of myocardial infarction in rats}

All experiments and methods were carried out following the IACUC guidelines. The experimental procedures were approved by the Animal Care Committee of the University of Hong Kong. Prior to animal experiments, PACA was dissolved in $0.9 \%$ saline containing $5 \%$ ethanol as a stock solution $(150 \mathrm{mg} / \mathrm{ml})$. Thirty-two male Sprague-Dawley rats were randomly assigned to Sham group and surgery group in 1:3 ratio. Rat model of myocardial ischemia reperfusion was induced by the ligation of the left anterior descending (LAD) artery. In practical, the surgery procedure was carried out as follows: rats were anesthetized with ketamine $(90 \mathrm{mg} / \mathrm{kg})$ plus xylazine $(10 \mathrm{mg} / \mathrm{kg})$ and ventilated through a small animal volume-control ventilator at 95 breaths and $1.8 \mathrm{~mL}$ volume. The chest was opened between the $3^{\text {rd }}$ and $4^{\text {th }}$ rib. Following the thoracotomy, rat hearts were exposed, and the LAD artery was subsequently ligated with a 6-0 silk suture. At the end of surgery, the chest was closed. Rats in sham group were subjected to the same surgical procedures except for LAD artery ligation. For drug treatment, at $24 \mathrm{~h}$ after LAD artery ligation, MI group received i.p. injection of vehicle ( $0.5 \mathrm{ml} /$ day) for 3, 5 or 7 days; MI+PACA group received i.p. injection of PACA (15 mg/ $\mathrm{kg}$ /day) for 3, 5 or 7 days; Sham group received i.p. injection of vehicle ( $0.5 \mathrm{ml} /$ day) for 3, 5 or 7 days; At the end of experiments, $1 \%$ Evans blue dye was injected and rat hearts were quickly collected, frozen, and cut into $2 \mathrm{~mm}$-sections. Five sections from each rat were incubated in $1 \%$ TTC at $37^{\circ} \mathrm{C}$ for $15 \mathrm{~min}$, and fixed in $4 \%$ formalin solution. The infarct area (IA, white color or yellow color) and area at risk (AAR, red, white and yellow color) were assessed. AAR and IA were measured by computerized planimetry using NIH Image J software.

\section{Histopathological examination}

The histopathological examination was conducted as previously described [32]. In brief, at the end of drug treatment as indicated, animals were euthanized. Cardiac samples were collected and fixed in $4 \%$ paraformaldehyde in PBS (Sigma, St. Louis, MO, USA). Paraffin-embedded sections (5- $\mu \mathrm{m})$ were prepared and stained with hematoxylin and eosin (H\&E) stain. H\&E stained sections were imaged and examined under light microscope for gross myocyte injury and the effect of interventions

\section{Immunohistochemistry staining}

Heart tissues were embedded in paraffin wax and processed into 5 - $\mu \mathrm{m}$ thin slices. Following deparaffinization and rehydration, tissue sections were subjected to antigen retrieval in $0.01 \mathrm{M}$ citrate buffer ( $\mathrm{pH}$ 6.0) in a microwave oven. Tissue sections were subsequently blocked with 5\% goat serum in TBS-T buffer, and probed with primary antibodies against ED1 and ED2 overnight. The antibodies retained on the sections were detected with the corresponding secondary antibodies at room temperature for 90 min. The slides were imaged on a Zeiss microscopy (Carl-Zeiss, Jena, Germany). The ED1 and ED2 positive cells were counted from five different pictures from one slide. And the results were derived from three heart tissue slides from three different rats.

\section{Statistical analysis}

The results were presented as means \pm SD or means \pm SEM from three or more independent experiments. Statistical analysis was performed by one-way analysis of variance (ANOVA) with post hoc Fisher's least significant difference (LSD) test, Dunnett's test or Student's t-test using SPSS software 20.0. A $p$-value of $<0.05$ was considered as statistically significant. 


\section{Results}

Caffeic acid derivatives differently regulated the expression of iNOS and COX-2 against LPS challenge

We initially examined the effects of caffeic acid (CA) and four derivatives on the growth of RAW264.7 macrophages. The IC50 concentrations of these compounds were calculated as follows: $642.5 \mu \mathrm{M}, \mathrm{CA} ; 262.0 \mu \mathrm{M}$, PACA; $92.7 \mu \mathrm{M}$, PCA; $54.2 \mu \mathrm{M}$, PCE and 10.6 $\mu \mathrm{M}, \mathrm{CAPE}$. We selected the sub-toxic concentration of $10 \mu \mathrm{M}$ for these caffeic acid compounds for the treatment of cell culture. RAW264.7 macrophages were pre-treated with caffeic acid compounds and challenged with LPS. We examined the effects of five compounds on the induction of iNOS and COX-2 in LPS-stimulated RAW264.7 macrophages by Western blotting. As shown in Fig. 1A. PCE, PCA and PACA showed strong inhibitory effects on iNOS induction and no much effects on COX-2 induction against LPS stimulation. As an exception, CAPE abolished the stimulatory effects of on the induction of iNOS and COX-2 expression. By taking the potency and cytotoxicity into account, we further examined whether the concentrations (e.g., 0, 10, 20 and $50 \mu \mathrm{M}$ ) of PACA affected the expression of iNOS and COX-2 in LPS-stimulated

Fig. 1. Differential effects of caffeic acid derivatives on the expression of macrophage M1 and M2 biomarkers. (A) Caffeic acid derivatives differentially regulated the expression of iNOS and COX-2 in LPS-stimulated macrophages. RAW264.7 cells were subjected to pre-treatment with $10 \mu \mathrm{M}$ caffeic acid derivatives for $2 \mathrm{~h}$ and subsequent challenge with $1 \mu \mathrm{g} /$ $\mathrm{mL}$ of LPS for $24 \mathrm{~h}$. The levels of iNOS and COX-2 were detected by Western blotting and quantified by NIH Image J software (http:// imagej.nih.gov/ij/). The results were expressed as Mean \pm SD $(\mathrm{n}=3)$. \#\#, $\mathrm{p}<0.01$ (LPS alone vs untreated control); b, $\mathrm{p}<0.01$ (Drug treatment vs LPS alone). (B) PACA selectively suppressed

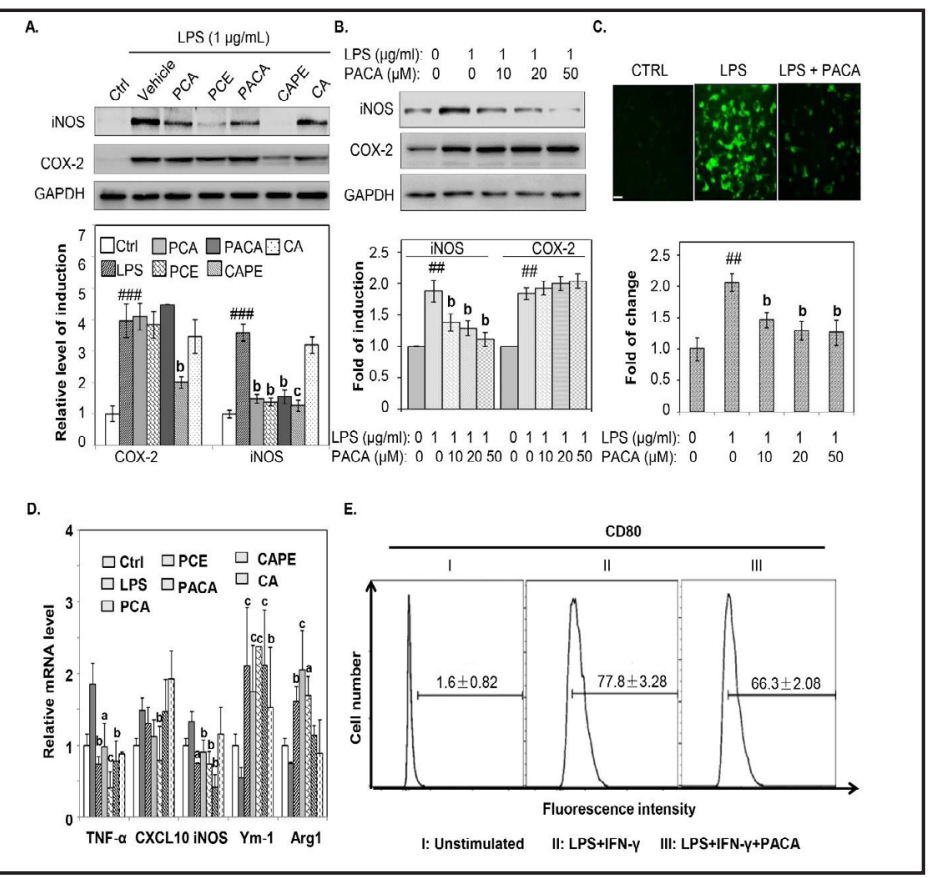
LPS-induced iNOS over COX-2. The experiments were performed as described in Panel A. The results were expressed as Mean \pm SD ( $n=3)$. \#\#, $\mathrm{p}<0.01$ (LPS alone vs untreated control); $b, \mathrm{p}<0.01$ (Drug treatment vs LPS alone). (C) Effects of PACA on LPSinduced NO production. The cells were subjected to pre-treatment with PACA at indicated concentrations for $2 \mathrm{~h}$ and subsequent challenge with $1 \mu \mathrm{g} / \mathrm{mL}$ of LPS for $24 \mathrm{~h}$. The NO contents were determined by staining with DAF-AM acetate and analyzed under a fluorescence microscopy. The images were analyzed with NIH Image J software. The results were expressed as the percentage of the untreated control $(n=3)$. \#\#, $\mathrm{p}<0.01$, (LPS only vs untreated control); $b, p<0.01$, (Drug treatment vs LPS alone). (D) Effects of caffeic acid derivatives on the mRNA expression of macrophage biomarkers. RAW264.7 macrophages were cultured in DMEM with 10\% FBS, and then pretreat with caffeic acid derivatives for $2 \mathrm{~h}$, and then stimulated with $1 \mu \mathrm{g} / \mathrm{mL}$ LPS for another $24 \mathrm{~h}$. M1 biomarkers (i.e., TNF- $\alpha$, iNOS and CXCL10) and M2 biomarkers (i.e., Arg1 and Ym-1) were analyzed by RT-PCR. a, P<0.05; b, P<0.01; c, P<0.001 (Drug + LPS vs LPS alone). (E) Flow cytometric analysis of macrophage M1 biomarker CD80. Following the treatment with indicated drugs as described in Experimental methods, RAW264.7 macrophages were stained with fluorophore-labeled antibodies against CD80, and analyzed by flow cytometry on a BD LSR Fortessa Cell Analyzer. The data were analyzed using FlowJo software. The results were expressed as a percentage of the untreated control $(n=3)$. 
Cheng et al.: Targeting Macrophages for Resolution of Inflammation

RAW264.7 cells. As shown in Fig. 1B, PACA selectively attenuated the stimulatory effects of LPS on the expression of iNOS in a concentration-dependent manner but did not change COX-2 expression. Consistently, PACA dramatically diminished LPS-induced production of the intracellular NO species in a concentration-dependent manner (Fig. 1C).

\section{Caffeic acid derivatives altered the expression profiles of macrophage M1 and M2 biomarkers}

To investigate the effects of caffeic acid derivatives on macrophage polarization, we determined several well-recognized macrophage biomarkers by RT-PCR or flow cytometric analysis. As shown in Fig. 1D, LPS increased the mRNA levels of M1 biomarkers (e.g., TNF- $\alpha$, CXCL10 and iNOS) in RAW264.7 macrophages. PACA, PCA and PCE effectively suppressed the upregulation of the mRNAs for M1 biomarker against LPS stimulation. PACA showed the strongest inhibition, whereas CA and CAPE exhibited much less activity. On the other hand, PACA markedly upregulated the expression of Ym-1 mRNA and Arg1 mRNA, whereas PCA, PCE, CAPE and CA failed to increase the expression of these M2 biomarker mRNAs. We further verified the effect of PACA on the expression of M1 biomarker CD80 by flow cytometry. Fig. $1 \mathrm{E}$ showed that PACA effectively reduced the population of $\mathrm{CD} 80^{+} \mathrm{M} 1$ macrophages from $77.8 \%$ to $66.3 \%$ in response to LPS and IFN- $\gamma$.

\section{Caffeic acid derivatives inhibited macrophage chemotaxis}

To examine the effects of caffeic acid derivatives on the functions of macrophages, we first investigated the effects of caffeic acid derivatives on macrophage chemotaxis towards apoptotic cardiomyocytes by Transwell migration assay as previously described [33]. Rat cardiomyocyte H9c2 cells were challenged under oxygen glucose deprivation (OGD) conditions to undergo apoptosis. The cell culture medium was collected as the conditioned medium and placed in the lower chambers in a Transwell plate. Following the treatment

Fig. 2. Effects of caffeic acid derivatives on the migration and phagocytosis of macrophages. (A) Effects of caffeic acid derivatives on the chemotactic potential of RAW264.7 macrophages. After the treatment with caffeic acid derivatives and LPS for $24 \mathrm{~h}$, the cell culture medium was collected as the conditioned medium for the assay of chemotactic potential in Transwell plates.

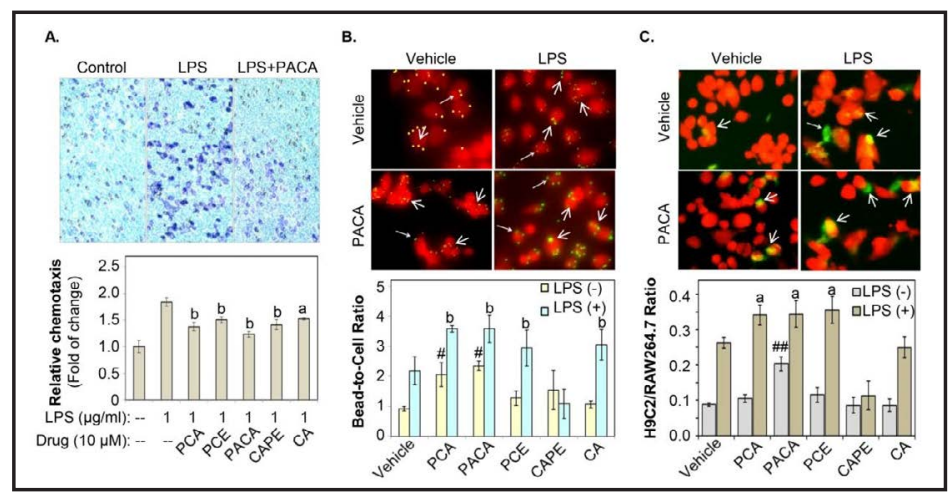
Fresh macrophages that migrated towards the conditioned medium were fixed, stained and imaged under a microscope. Representative images were shown. Macrophage migration was quantified by measuring the absorbance of cell extracts as described in "Experimental methods". The results were expressed as Means \pm SD $(n=3) . a, p<0.05 ; b$, $\mathrm{p}<0.01$ (Drug + LPS vs LPS alone); \#, p<0.05 (Drug vs vehicle). (B) Effects of caffeic acid derivatives on the phagocytosis of latex beads by macrophages. After $24 \mathrm{~h}$ treatment with caffeic acid derivatives and LPS, RAW264.7 macrophages were stained with CellTracker ${ }^{\mathrm{TM}}$ Orange CMTMR dye, and subsequently incubated with FITC-labeled beads for $1.5 \mathrm{~h}$. The images were acquired on a fluorescence microscope and analyzed with NIH Image J software. (C) Effects of caffeic acid derivatives on the phagocytosis of apoptotic H9c2 cells. After the treatment with caffeic acid derivatives and LPS for 24 h, RAW264.7 macrophages were labeled with CellTracker ${ }^{\mathrm{TM}}$ Orange CMTMR dye. H9C2 cells were driven to undergo apoptosis by OGD exposure for $12 \mathrm{~h}$ and labeled with CellTracker ${ }^{\mathrm{TM}}$ Green CMTMR dye. After labeling, RAW264.7 macrophages and apoptotic H9C2 cells were incubated for $1.5 \mathrm{~h}$. The images were acquired under a fluorescence microscope and analyzed with NIH Image J software. a, p<0.05; b, p<0.01 (Drug + LPS vs LPS alone); \#\#, p<0.01 (Drug vs vehicle).

\section{KARGER}


with caffeic acid derivatives and LPS as described in Methods, RAW264.7 macrophages were evaluated for migration through Transwell membrane from the upper chamber. By counting macrophages that migrated through Transwell membrane, as shown in Fig. 2A, LPS enhanced macrophage migration by up to 1.8 -fold compared with untreated control. Caffeic acid derivatives effectively attenuated the stimulatory effect of LPS on macrophage chemotaxis. Particularly, PACA showed the strongest inhibition on LPS-induced chemotaxis of macrophages.

\section{Caffeic acid derivatives enhanced macrophage phagocytosis}

As for macrophage functions, we subsequently examined the effect of caffeic acid derivatives on macrophage phagocytosis. The first experiment was to assay the phagocytosis of latex beads. RAW264.7 cells were labeled with CellTracker ${ }^{\mathrm{TM}}$ Orange CMTMR probe prior to the incubation with FITC-labeled latex beads with the size of $1.0 \mu \mathrm{m}$. As shown in Fig. 2B, PACA and PCA promoted the binding of latex beads onto macrophages and further enhanced the stimulatory effects of LPS on macrophage phagocytosis. PCE and CA only enhanced the effects of LPS on macrophage phagocytosis. CAPE did not show much effect on macrophage phagocytosis possibly due to its cytotoxicity. The second experiment was to assay the phagocytosis of apoptotic cardiomyocytes. H9c2 cells were challenged under OGD conditions to induce apoptosis. Apoptotic H9c2 cells were subsequently labeled with CellTracker ${ }^{\mathrm{TM}}$ Green CMTMR probe whereas RAW264.7 macrophages stained with CellTracker ${ }^{\mathrm{TM}}$ Orange CMFDA probe after drug treatment. As shown in Fig. 2C, PACA not only increased the binding of H9c2 cells to macrophages and but also enhanced the effects of LPS on macrophage phagocytosis. Other drugs except for CAPE only enhanced the effects of LPS on macrophage phagocytosis.

Fig. 3. In vivo cardioprotective effects of PACA against myocardial ischemia injury. (A) Experimental design. Rats were randomly divided into three groups: Sham, LAD and LAD+ PACA PACA was administered via i.p. injection at the dose of $15 \mathrm{mg} / \mathrm{kg} /$ day at $24 \mathrm{~h}$ after LAD artery ligation, whereas vehicle with equal volume was administered to Sham and Model animals. On Day 3, Day 5 and Day 7, the heart tissues were collected for analysis as described in "Experimental methods". (B) Assessment of infarct size. (C) Histopathological examination of rat hearts. The cardiac tissues from three rats

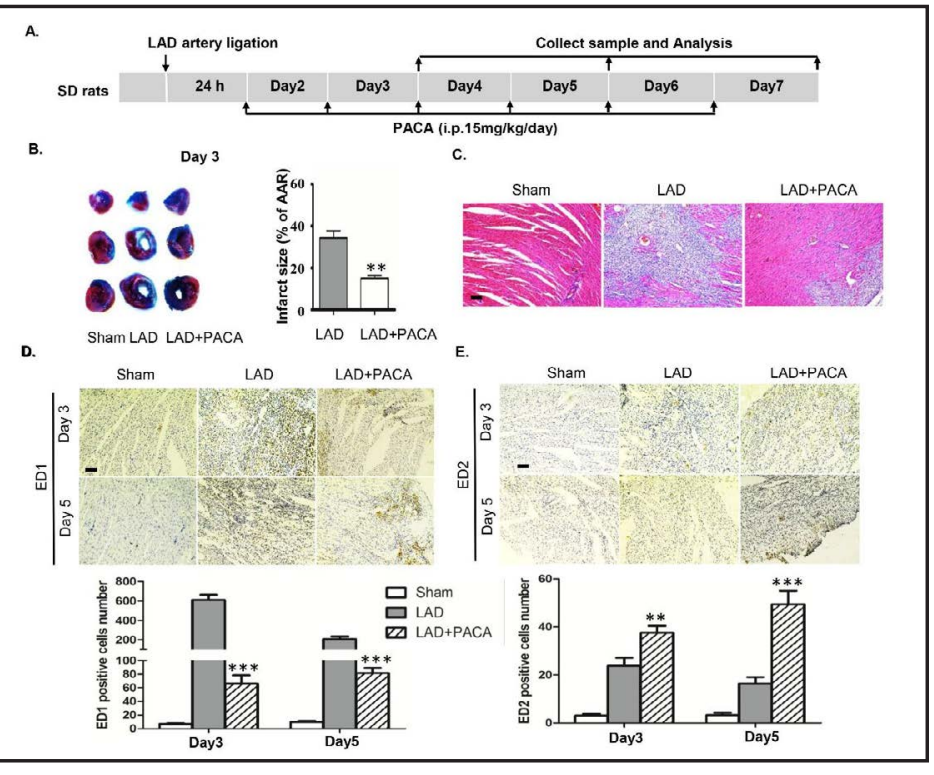
per group were stained with

H\&E stain. Representative images were selected from four groups. (D) Immunohistochemical detection of ED1 positive cells in infarcted myocardium. The cardiac tissues were probed with anti-ED1 antibody and visualized by immunohistochemical approach. The images were captured under a microscope. The ED1 positive cells from five different pictures were counted and analyzed by GraphPad Prism. ***, p<0.001. (E) Immunohistochemical detection of ED2 positive cells in infarcted myocardium. The cardiac tissues were probed with anti-ED2 antibody and visualized by immunohistochemical approach. The images were captured under a microscope. The ED2 positive cells from five different pictures were counted and analyzed by GraphPad Prism. **, p<0.01; ***, p<0.001. Scale bar, $100 \mu \mathrm{m}$.

\section{KARGER}


PACA protected hearts against ischemia-induced cardiac injury

To evaluate the in vivo cardioprotective efficacy of PACA, we induced myocardial infarction in rats by ligating LAD artery as previously described [34]. PACA was administered via i.p. injection at $24 \mathrm{~h}$ after artery ligation. Based on TTC staining in Fig. 3B, PACA reduced myocardial infarct size on Day 3 from $34.5 \%$ to $15.0 \%$. As shown in Fig. 3C, H\&E staining identified the disruption of myofibrillar structure and the accumulation of neutrophil/ macrophage clusters in the ischemic myocardium. PACA at the dose of $15 \mathrm{mg} / \mathrm{kg}$ not only preserved myofibrillar structure but also reduced the recruitment of inflammatory cells and myolysis.

\section{PACA skewed macrophage polarization from M1 to M2 phenotype in infarcted hearts}

To define the phenotypes of cardiac macrophages, we stained macrophage M1 biomarker ED1 and M2 biomarker ED2 with specific antibodies. As shown in Fig. 3D and 3E, ED1 ${ }^{+}$M1 macrophages were barely detectable in control group, but markedly increased in MI group. PACA effectively decreased the number of ED1 positive cells. On the other hand, ED2 ${ }^{+} \mathrm{M} 2$ macrophages were barely detected in control group as well as MI group. PACA effectively increased the number of ED2 ${ }^{+} \mathrm{M} 2$ macrophages.

\section{PACA did not attenuate LPS-induced activation of MAP kinases}

To explore the potential mechanisms, we first examined the effect of PACA on LPSinduced activation of MAP kinases (e.g., ERK1/2, p38 and JNK). As shown in Fig. 4A, LPS dramatically induced the phosphorylation of ERK1/2, P38 and JNK. PACA barely affected such activities of LPS against MAP kinases.

Fig. 4. Elucidation of the potential mechanisms underlying the biological activities of PACA against LPS challenge. (A) Effects of PACA against LPS-induced activation of MAP kinases. The cells were treated with PACA at indicated concentrations for 2 $h$, and challenged with LPS for $30 \mathrm{~min}$. The levels of total and phosphorylated ERK1/2, p38 and JNK were detected by Western blotting. (B) Immunostaining of intracellular NF-kB p65 subunit. The cells were treated with $20 \mu \mathrm{M}$ PACA for $2 \mathrm{~h}$, and challenged with

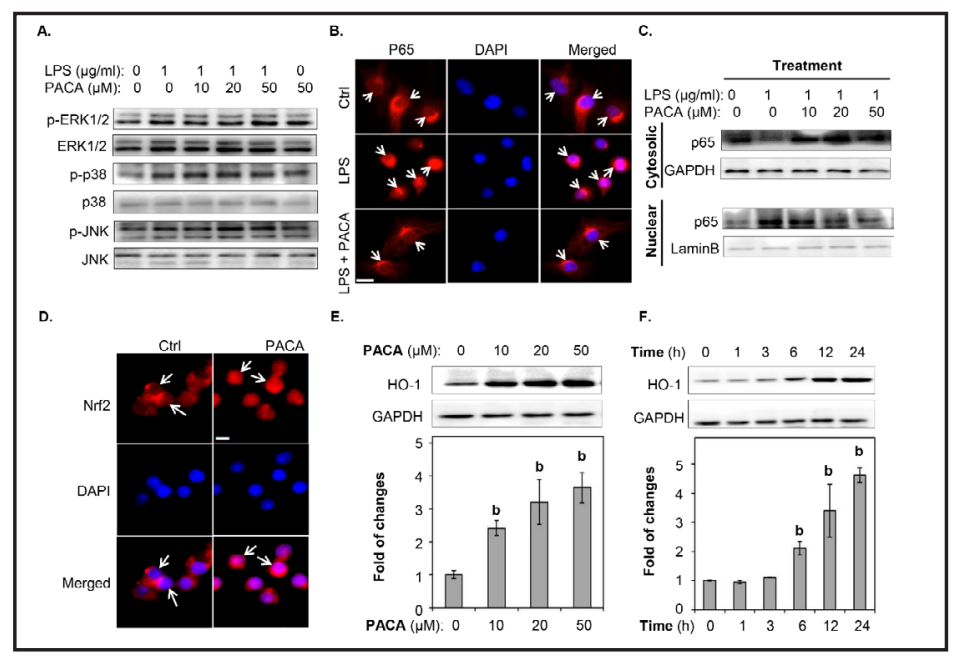
LPS for $6 \mathrm{~h}$. NF-kB p 65 subunit was detected by immunostaining with p65 antibody, whereas the nuclei were stained by DAPI. Images were acquired under a fluorescence microscopy. (C) Western blot analysis of NF- $\kappa$ B p65 nuclear translocation. After drug treatment, the nuclear proteins were isolated and detected by Western blotting with antibody against NF-kB p65 subunit. (D) Immunostaining of intracellular Nrf2. After 6 h treatment with $20 \mu \mathrm{M}$ PACA, the intracellular Nrf2 was detected by immunostaining with specific antibody, whereas the nuclei were detected by DAPI staining. (E) Concentration dependence in PACA-induced HO-1 expression. After $24 \mathrm{~h}$ incubation with PACA at different concentrations, the expression of HO-1 was examined by Western blotting and quantified by a densitometric method. (F) Time course of HO-1 induction by PACA. Cells were treated with $20 \mu \mathrm{M}$ PACA for $0,1,3,6,12$ and $24 \mathrm{~h}$, the expression of HO-1 was examined by Western blotting and quantified by a densitometric method. The results were expressed as a percentage of the untreated control $(\mathrm{n}=3)$. a, p <0.05, b, p<0.01 (Sample vs control). 
PACA exhibited the anti-inflammatory activities via activating the Nrf2/HO-1 pathway

To study the regulation of iNOS induction, we tentatively investigated the effects of PACA on LPS-induced activation of NF- $\kappa$ B pathway. By immunostaining and Western blotting of NFkB p65 subunit, we found that PACA effectively inhibited LPS-induced nuclear translocation of NF-kB p65 subunit (as shown in Fig. 4B and 4C). We focused on the effects of PACA on the Nrf2/HO-1 pathway by immunostaining and Western blot analysis with specific antibodies. Following the treatment with PACA, the intracellular Nrf2 in macrophages was probed with Nrf2 antibody. As indicated in Fig. 4D, PACA strongly induced the nuclear translocation of Nrf2. Western blot analysis in Fig. 4E and 4F also showed that PACA induced HO-1 expression in a concentration and time-dependent manners.

To clarify the impact of HO-1 induction on the anti-inflammatory activities of PACA, we employed HO-1 specific inhibitor Sn(IV) protoporphyrin IX dichloride (SnPP) to block the enzyme activity of HO-1, and subsequently examined NF-kB activation, iNOS induction and HSC recruitment. Following the treatment with LPS alone or in combination with PACA and SnPP, macrophages were probed with antibody against NF-kB p65 subunit. As shown in Fig. 5A and 5B, HO-1 inhibitor SnPP attenuated the inhibitory effect of PACA on LPSinduced nucleus translocation of NF-kB p65 subunit. Western blot analysis in Fig. 5C and 5D suggested that HO-1 inhibitor dramatically recovered LPS-induced expression of iNOS and COX-2. PACA effectively attenuated LPS-induced expression of iNOS over COX-2. HO-1 inhibitor SnPP almost abolished the effect of PACA on LPS-induced expression of iNOS but showed no activity against COX-2 expression.

\section{Discussion}

Our previous studies not only synthesized PACA and PCA but also evaluated several caffeic acid derivatives for cardioprotective, neuroprotective and neurotrophic activities

Fig. 5. Regulation of the crosstalks between $\mathrm{NF}-\kappa \mathrm{B}$ and $\mathrm{Nrf2/}$ HO-1 pathways by PACA and the relevance to the induction of iNOS and COX-2. (A) PACA suppressed $\mathrm{NF}-\mathrm{kB}$ activation via inducing $\mathrm{HO}-1$. Cells were pre-treated with $20 \mu \mathrm{M}$ PACA with or without $20 \mu \mathrm{M} \mathrm{HO}-1$ inhibitor SnPP and challenged with LPS for $6 \mathrm{~h}$. Intracellular p65 subunit was examined by immunostaining with specific antibody. (B) Western blot analysis of NF- $\kappa$ B p65 nuclear translocation in the presence of HO-1 inhibitor. After drug treatment, the nuclear

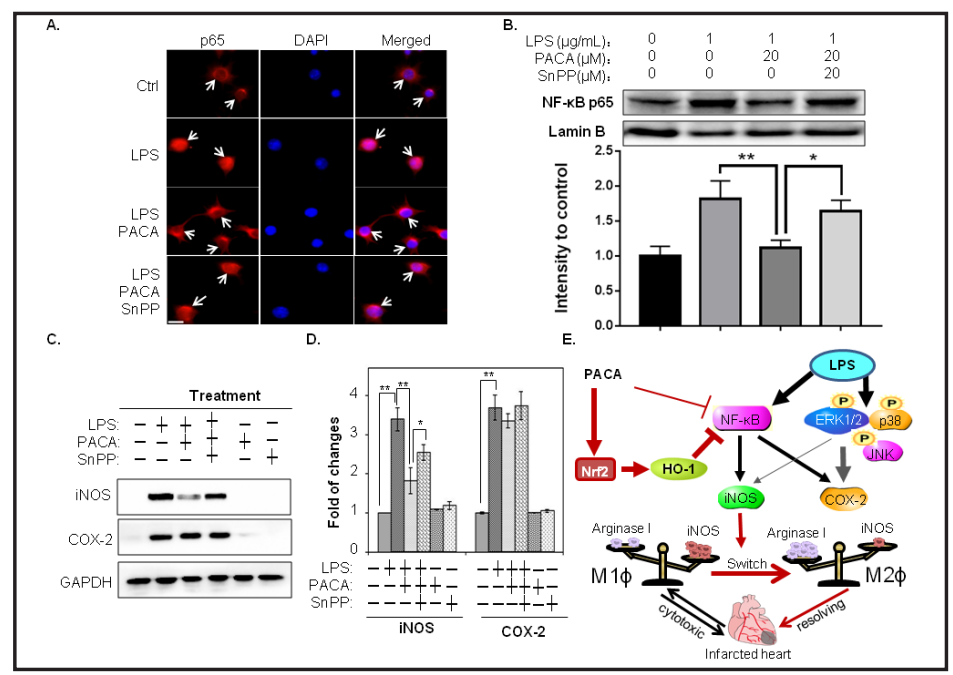
proteins were isolated and detected by Western blotting with antibody against NF- $\mathrm{kB}$ p65 subunit. The blots were quantified by a densitometric method. $\mathrm{a}, \mathrm{P}<0.05 ; \mathrm{b}, \mathrm{P}<0.01$. (C and D) PACA selectively inhibited iNOS expression over COX-2 via inducing HO-1. After the treatments as described in Panel A, the levels of iNOS and COX-2 were examined by Western blotting analysis. a, $\mathrm{P}<0.05$; b, $\mathrm{P}<0.01$. (E) Proposed mechanisms by which PACA promotes macrophage M2 polarization and protects hearts against ischemia-induced injury. PACA activates Nrf2/ HO-1 pathway and inhibits NF- $\kappa B$ pathway without affecting MAP kinases. Consequently, PACA selectively suppresses the induction of iNOS over COX-2 expression against LPS stimulation. Ultimately, PACA promotes M2 macrophage polarization for the resolution of ischemia-induced inflammation. 
$[19,26]$. The aim of the present study was to further examine the effects of caffeic acid derivatives on the phenotypes and functions macrophages in myocardial infarction. Our pilot studies suggested that PACA and analogs differentially attenuated iNOS expression over COX-2 expression in LPS-stimulated macrophages. Thus, we hypothesized that PACA might regulate macrophage polarization against myocardial infarction. Our work led to three key findings as follows: 1) PACA effectively promoted macrophage polarization towards a resolving M2-phenotype; 2) PACA reduced myocardial injury in a rat model of myocardial infarction.; 3) PACA mainly regulated two key redox sensitive signaling pathways, namely Nrf2/HO-1 pathway and NF-kB pathway.

Inflammation is an important therapeutic target for the treatment of myocardial infarction [1]. The existing anti-inflammatory drugs are mainly designed to target the production of proinflammatory mediators such as NO, PGE2, TXA2, TNF- $\alpha$ and IL-1 $\beta$ via regulating ERK, p38, JNK, NF-kB and Nrf2 [35-37]. In the present study, we found that PACA exhibited selective activity against iNOS up-regulation and NO overproduction in LPSstimulated macrophages, while showed no activity against COX-2 expression. To elucidate the underlying mechanisms, we firstly investigated the effects of PACA on the activation of MAP kinases (i.e., ERK, p38 and JNK) and the nuclear translocation of NF-kB. Surprisingly, PACA did not affect the phosphorylation of ERK, p38 and JNK. LPS and many other proinflammatory stimuli are known to activate the NF- $\mathrm{KB}$ pathway for regulating the expression of immunomodulating and inflammatory genes [38]. NF- $\kappa B$ activation is a complex process involving rapid phosphorylation and degradation of IKB. Ultimately, NF-kB p65 and p50 subunits are translocated to the cell nucleus and induce iNOS and COX-2 in an equally effective manner $[39,40]$. Based on Western blotting and immunostaining, we found that PACA reduced the nuclear translocation of NF-kB in LPS-stimulated macrophages. These results suggest that inhibition of NF- $\kappa B$ pathway is only part of the mechanisms by which PACA selectively suppresses the expression of iNOS over COX-2.

To further clarify the selective effects of PACA on iNOS expression, we focused on the antioxidant Nrf2/HO-1 pathway. Nrf2 is a redox sensitive transcriptional factor and regulates the cellular defense against oxidative stress [41]. Oxidative stressors and some anti-inflammatory electrophilic drugs disrupt Keap1-Nrf2 complex, promote the nuclear translocation of Nrf2 and finally activate the transcription of antioxidant genes including HO-1 [42]. We have previously identified several botanicals (e.g., Z-ligustilide, senkyunolide $\mathrm{H}$, senkyunolide I, puerarin, PACA) for activating the Nrf2-Keap1 pathway and inducing HO-1 expression [18-20, 43]. In the present study, therefore, we hypothesized that PACA might regulate iNOS induction and macrophage M2 polarization via activating Nrf2/HO-1 pathway. HO-1 is well-known to orchestrate the cross-talks between Nrf2 and NF-kB pathways [14, $44,45]$. Thus, we further examined whether HO-1 inhibitor SnPP could affect the activity of PACA. Indeed, PACA induced HO-1 expression in RAW264.7 macrophages in a concentrationand time-dependent manner. HO-1 specific inhibitor SnPP diminished the inhibitory effect of PACA on NF- $\kappa B$ activation and iNOS induction in LPS-stimulated macrophages. These results suggest that PACA activates Nrf2/HO-1 as the key mechanism for inhibiting NF- $\mathrm{KB}$ pathway and subsequently down-regulating LPS-induced iNOS expression.

The overexpression of iNOS is strongly associated with M1 macrophage polarization [46]. In the present study, we identified the selectivity of caffeic acid derivatives (e.g., PACA, PCA and PCE) against LPS-induced iNOS expression over COX-2 expression. We further clarified whether caffeic acid derivatives could modulate the phenotypic and functional switch of macrophages. Our results demonstrated that PACA and other caffeic acid derivatives not only reduced the expression of M1 biomarkers (e.g., iNOS, TNF- $\alpha$, CXCL10) but also increased the expression of M2 biomarkers (e.g., Arg1, Ym-1). By detecting phenotypic biomarkers with flow cytometric analysis and tissue immunofluorescence, we confirmed that PACA could skew macrophage polarization towards a M2 phenotype. Others

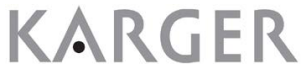


have already demonstrated that macrophage M1/M2 polarization is an important target for the regulation of inflammatory and immune responses [47-50]. Macrophage polarization was modulated by several signaling pathways, such as PKA-STAT3, TLR4 and so on [51, 52]. Thus, PACA may promoting macrophage M2 polarization for the resolution of inflammation, clearance of debris and tissue healing.

In conclusion, the present study demonstrated that PACA and other caffeic acid derivatives could promote macrophage polarization from proinflammatory M1 to antiinflammatory/resolving M2 phenotype. The present study also discovered that PACA might exhibit cardioprotective potential via activating Nrf2/HO-1 pathway and inhibiting NF- $\mathrm{BB}$ pathway (Fig. 5D). Therefore, we anticipate that PACA is a promising drug candidate for the therapy of myocardial infarction.

\section{Acknowledgements}

This work was supported by GRF grants $(17120915,17146216)$ from the Research Grants Council of Hong Kong Government (to J.R.) and two internal Seed Funds for Basic Research Programme from University of Hong Kong (201411159130, 201511159245).

Y.Y. Cheng, C.B. Yang and D. Luo performed experiments, analyzed data, and wrote the manuscript. X.C. Li and X.C. Le provided the technical support and discussed the results. J. Rong designed the research, supervised data analysis and wrote the article.

\section{Disclosure Statement}

The authors declare that they have no competing interests.

\section{References}

1 Yellon DM, Hausenloy DJ: Myocardial reperfusion injury. N Engl J Med 2007;357:1121-1135.

2 Dobaczewski M, Gonzalez-Quesada C, Frangogiannis NG: The extracellular matrix as a modulator of the inflammatory and reparative response following myocardial infarction. J Mol Cell Cardiol 2010;48:504-511.

- 3 Gordon S, Taylor PR: Monocyte and macrophage heterogeneity. Nat Rev Immunol 2005;5:953-964.

4 Novak ML, Koh TJ: Macrophage phenotypes during tissue repair. J Leukoc Biol 2013;93:875-881.

5 Kim YS, Kang WS, Kwon JS, Hong MH, Jeong HY, Jeong HC, Jeong MH, Ahn Y: Protective role of 5-azacytidine on myocardial infarction is associated with modulation of macrophage phenotype and inhibition of fibrosis. J Cell Mol Med 2014;18:1018-1027.

6 Martinez FO, Helming L, Gordon S: Alternative activation of macrophages: an immunologic functional perspective. Annu Rev Immunol 2009;27:451-483.

7 Xie QW, Kashiwabara Y, Nathan C: Role of transcription factor NF-kappa B/Rel in induction of nitric oxide synthase. J Biol Chem 1994;269:4705-4708.

-8 Gordon S, Martinez FO: Alternative activation of macrophages: mechanism and functions. Immunity 2010;32:593-604.

-9 Troidl C, Mollmann H, Nef H, Masseli F, Voss S, Szardien S, Willmer M, Rolf A, Rixe J, Troidl K, Kostin S, Hamm C, Elsasser A: Classically and alternatively activated macrophages contribute to tissue remodelling after myocardial infarction. J Cell Mol Med 2009;13:3485-3496.

10 Nahrendorf M, Pittet MJ, Swirski FK: Monocytes: protagonists of infarct inflammation and repair after myocardial infarction. Circulation 2010;121:2437-2445.

11 Naito Y, Takagi T, Higashimura Y: Heme oxygenase-1 and anti-inflammatory M2 macrophages. Arch Biochem Biophys 2014;564:83-88. 


\section{Cellular Physiology Cell Physiol Biochem 2018;47:2544-2557 and Biochemistry \begin{tabular}{l|l} 
DOI: 10.1159/000491651 & $\begin{array}{l}\text { C } 2018 \text { The Author(s). Published by S. Karger AG, Basel } \\
\text { www.karger.com/cpb }\end{array}$
\end{tabular}

12 Sierra-Filardi E, Vega MA, Sanchez-Mateos P, Corbi AL, Puig-Kroger A: Heme Oxygenase-1 expression in M-CSF-polarized M2 macrophages contributes to LPS-induced IL-10 release. Immunobiology 2010;215:788-795.

13 Gozzelino R, Jeney V, Soares MP: Mechanisms of cell protection by heme oxygenase-1. Annu Rev Pharmacol 2010;50:323-354.

14 Paine A, Eiz-Vesper B, Blasczyk R, Immenschuh S: Signaling to heme oxygenase-1 and its anti-inflammatory therapeutic potential. Biochem Pharmacol 2010;80:1895-1903.

15 Onyiah JC, Sheikh SZ, Maharshak N, Steinbach EC, Russo SM, Kobayashi T, Mackey LC, Hansen JJ, Moeser AJ, Rawls JF, Borst LB, Otterbein LE, Plevy SE: Carbon monoxide and heme oxygenase-1 prevent intestinal inflammation in mice by promoting bacterial clearance. Gastroenterology 2013;144:789-798.

16 Pae HO, Lee YC, Chung HT: Heme oxygenase-1 and carbon monoxide: emerging therapeutic targets in inflammation and allergy. Recent Pat Inflamm Allergy Drug Discov 2008;2:159-165.

17 Motterlini R, Haas B, Foresti R: Emerging concepts on the anti-inflammatory actions of carbon monoxidereleasing molecules (CO-RMs). Med Gas Res 2012;2:28.

18 Zhao J, Cheng YY, Fan W, Yang CB, Ye SF, Cui W, Wei W, Lao LX, Cai J, Han YF, Rong JH: Botanical drug puerarin coordinates with nerve growth factor in the regulation of neuronal survival and neuritogenesis via activating ERK1/2 and PI3K/Akt signaling pathways in the neurite extension process. CNS Neurosci Ther 2015;21:61-70.

19 Yang C, Zhao J, Cheng Y, Le XC, Rong J: N-Propargyl Caffeate Amide (PACA) Potentiates Nerve Growth Factor (NGF)-Induced Neurite Outgrowth and Attenuates 6-Hydroxydopamine (6-OHDA)-Induced Toxicity by Activating the Nrf2/HO-1 Pathway. ACS Chem Neurosci 2015;6:1560-1569.

20 Qi H, Han Y, Rong J: Potential roles of PI3K/Akt and Nrf2-Keap1 pathways in regulating hormesis of Z-ligustilide in PC12 cells against oxygen and glucose deprivation. Neuropharmacology 2012;62:16591670 .

-21 Gautam R, Jachak SM: Recent Developments in Anti-Inflammatory Natural Products. Med Res Rev 2009;29:767-820.

22 Olthof MR, Hollman PC, Katan MB: Chlorogenic acid and caffeic acid are absorbed in humans. J Nutr 2001;131:66-71.

23 Silva T, Oliveira C, Borges F: Caffeic acid derivatives, analogs and applications: a patent review (2009-2013). Expert Opin Ther Pat 2014;24:1257-1270.

24 Ho YJ, Lee AS, Chen WP, Chang WL, Tsai YK, Chiu HL, Kuo YH, Su MJ: Caffeic acid phenethyl amide ameliorates ischemia/reperfusion injury and cardiac dysfunction in streptozotocin-induced diabetic rats. Cardiovasc Diabetol 2014;13:98.

-25 Ozer MK, Parlakpinar H, Acet A: Reduction of ischemia--reperfusion induced myocardial infarct size in rats by caffeic acid phenethyl ester (CAPE). Clin Biochem 2004;37:702-705.

26 Cheng Y, Luo D, Xia Z, Tse H-F, Li X, Rong J: In vivo cardioprotective effects and pharmacokinetic profile of N-propyl caffeamide (PCA) against ischemia reperfusion injury. Archivum Immunologiae et Therapiae Experimentalis 2017;65:1-12

-27 Yang C, Li X, Rong J: Amygdalin isolated from Semen Persicae (Tao Ren) extracts induces the expression of follistatin in HepG2 and C2C12 cell lines. Chin Med 2014;9:23.

-28 Cheng Y, Zhao J, Tse HF, Le XC, Rong J: Plant Natural Products Calycosin and Gallic Acid Synergistically Attenuate Neutrophil Infiltration and Subsequent Injury in Isoproterenol-Induced Myocardial Infarction: A Possible Role for Leukotriene B4 12-Hydroxydehydrogenase? Oxid Med Cell Longev 2015;2015:434052.

29 Yang C, Zhao J, Cheng Y, Li X, Rong J: Bioactivity-guided fractionation identifies amygdalin as a potent neurotrophic agent from herbal medicine Semen Persicae extract. Biomed Res Int 2014;2014:306857.

-30 Xu Y, Feng L, Wang S, Zhu Q, Zheng Z, Xiang P, He B, Tang D: Calycosin protects HUVECs from advanced glycation end products-induced macrophage infiltration. J Ethnopharmacol 2011;137:359-370.

31 Wang G, Siow YL, O K: Homocysteine induces monocyte chemoattractant protein-1 expression by activating NF-kappaB in THP-1 macrophages. Am J Physiol Heart Circ Physiol 2001;280:H2840-2847.

32 Gupta P, Kanwal A, Putcha UK, Bulani Y, Sojitra B, Khatua TN, Kuncha M, Banerjee SK: Cardioprotective effect of ritonavir, an antiviral drug, in isoproterenol induced myocardial necrosis: a new therapeutic implication. J Transl Med 2013;11:80. 


\section{Cellular Physiology Cell Physiol Biochem 2018;47:2544-2557 \begin{tabular}{ll|l} 
and Biochemistry & $\begin{array}{l}\text { DOI: 10.1159/000491651 } \\
\text { Published online: July 10, } 2018\end{array}$ & $\begin{array}{l}\text { @ } 2018 \text { The Author(s). Published by S. Karger AG, Basel } \\
\text { www.karger.com/cpb }\end{array}$ \\
\hline
\end{tabular}}

-33 Schrijvers DM, De Meyer GR, Kockx MM, Herman AG, Martinet W: Phagocytosis of apoptotic cells by macrophages is impaired in atherosclerosis. Arterioscler Thromb Vasc Biol 2005;25:1256-1261.

-34 George JC, Liner A, Hoit BD: Isoproterenol-induced myocardial injury: a systematic comparison of subcutaneous versus intraperitoneal delivery in a rat model. Echocardiography 2010;27:716-721.

-35 Biswas SK, Sica A, Lewis CE: Plasticity of macrophage function during tumor progression: Regulation by distinct molecular mechanisms. Journal of Immunology 2008;180:2011-2017.

-36 Kyriakis JM, Avruch J: Mammalian Mapk Signal Transduction Pathways Activated by Stress and Inflammation: A 10-Year Update. Physiol Rev 2012;92:689-737.

37 Santos A: Anti-inflammatory compounds of plant origin. Part II. Modulation of pro-inflammatory cytokines, chemokines and adhesion molecules. Planta Med 2004;70:93-103.

38 Liou HC: Regulation of the immune system by NF-kappa B and I kappa B. J Biochem Mol Biol 2002;35:537546.

39 Baker RG, Hayden MS, Ghosh S: NF- kB, inflammation, and metabolic disease. Cell Metabolism 2011;13:1122.

40 Ben-Neriah Y, Karin M: Inflammation meets cancer, with NF-kappaB as the matchmaker. Nat Immunol 2011;12:715-723.

41 Giudice A, Arra C, Turco MC: Review of molecular mechanisms involved in the activation of the Nrf2-ARE signaling pathway by chemopreventive agents. Methods Mol Biol 2010;647:37-74.

42 Magesh S, Chen Y, Hu L: Small Molecule Modulators of Keap1-Nrf2-ARE Pathway as Potential Preventive and Therapeutic Agents. Medic Res Reviews 2012;32:687-726.

-43 Qi HY, Siu SO, Chen Y, Han YF, Chu IK, Tong Y, Lau ASY, Rong JH: Senkyunolides reduce hydrogen peroxideinduced oxidative damage in human liver HepG2 cells via induction of heme oxygenase-1. Chemic-Biol Interact 2010;183:380-389.

44 Yang YC, Lii CK, Wei YL, Li CC, Lu CY, Liu KL, Chen HW: Docosahexaenoic acid inhibition of inflammation is partially via cross-talk between Nrf2/heme oxygenase 1 and IKK/NF-kappaB pathways. J Nutr Biochem 2013;24:204-212.

45 Koliaraki V, Kollias G: A new role for myeloid HO-1 in the innate to adaptive crosstalk and immune homeostasis. Adv Exp Med Biol 2011;780:101-111.

46 Martinez F0, Gordon S: The M1 and M2 paradigm of macrophage activation: time for reassessment. F1000Prime Rep 2014;6:13.

47 Fujiu K, Wang J, Nagai R: Cardioprotective function of cardiac macrophages. Cardiovasc Res 2014;102:232239.

48 Li F, Zhu XH, Yang YS, Huang L, Xu JH: TIPE2 Alleviates Systemic Lupus Erythematosus Through Regulating Macrophage Polarization. Cell Physiol Biochem 2016;38:330-339.

-49 Mosser DM, Edwards JP: Exploring the full spectrum of macrophage activation. Nat Rev Immunol 2008;8:958-969.

50 Luo HL, Chen J, Luo T, Wu FX, Liu JJ, Wang HF, Chen M, Li LQ, Li H: Downregulation of MacrophageDerived T-UCR uc. 306 Associates with Poor Prognosis in Hepatocellular Carcinoma. Cell Physiol Biochem 2017;42:1526-1539.

51 Wang N, Gao J, Jia M, Ma X, Lei ZX, Da F, Yan F, Zhang HA, Zhou Y, Li MK, He GH, Meng JR, Luo XX: Exendin-4 Induces Bone Marrow Stromal Cells Migration Through Bone Marrow-Derived Macrophages Polarization via PKA-STAT3 Signaling Pathway. Cell Physiol Biochem 2017;44:1696-1714.

52 Shi J, Li Q, Sheng MX, Zheng M, Yu MS, Zhang L: The Role of TLR4 in M1 Macrophage-Induced EpithelialMesenchymal Transition of Peritoneal Mesothelial Cells. Cell Physiol Biochem 2016;40:1538-1548. 